\title{
Dispersion of carbon nanotubes in aluminum improves radiation resistance
}

Kang Pyo So ${ }^{1}$, Di Chen ${ }^{2}$, Akihiro Kushima ${ }^{1}$, Mingda Li $^{1}$, Sangtae Kim ${ }^{1}$, Yang Yang ${ }^{1}$, Ziqiang Wang $^{1}$, Jong Gil Park ${ }^{3}$, Young Hee Lee ${ }^{3}$, Rafael I. Gonzalez ${ }^{4}$, Miguel Kiwi ${ }^{4}$, Eduardo M. Bringa ${ }^{5}$, Shao $\mathrm{Lin}^{2 *}$ and $\mathrm{Ju} \mathrm{Li}^{1 *}$

${ }^{1}$ Department of Nuclear Science and Engineering and Department of Materials Science and Engineering, Massachusetts Institute of Technology, Cambridge, Massachusetts 02139, USA.

${ }^{2}$ Department of Nuclear Engineering, Texas A\&M University, College Station, Texas 77845, USA.

${ }^{3}$ IBS Center for Integrated Nanostructure Physics, Institute for Basic Science (IBS), Sungkyunkwan University, 440-746, Republic of Korea.

${ }^{4}$ Departamento de Fisica, Facultad de Ciencias, Universidad de Chile, Casilla 653, Santiago, 7800024, Chile.

${ }^{5}$ Facultad de Ciencias Exactas y Naturales, Universidad Nacional de Cuyo, Mendoza, 5500, Argentina.

We can mass-produce metal / carbon nanotube (CNT) composites that show improved radiation tolerance. The $0.5 \mathrm{wt} \% \mathrm{Al+CNT}$ composite showed improved tensile strength without reduction of tensile ductility before radiation, and reduced void/pore generation and radiation embrittlement at high displacements per atom (DPA). Under helium ion irradiation up to 72 DPA, the 1D carbon nanostructures survive, while $\mathrm{sp}^{2}$ bonded graphene transform to $\mathrm{sp}^{3}$ tetrahedral amorphous carbon. Self-ion (Al) irradiation converts CNTs to a metastable form of $\mathrm{Al}_{4} \mathrm{C}_{3}$, but still as slender $1 \mathrm{D}$ nanorods with prolific internal interfaces that catalyze recombination of radiation defects, reducing radiation hardening and porosity generation. The 1D fillers may also form percolating paths of "nano-chimneys" that outgas the accumulated helium and other fission gases, and provide an essential solution to the gas accumulation problem.

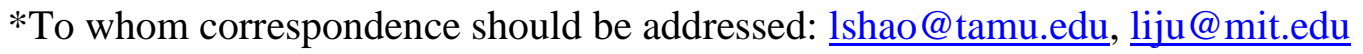


Nuclear fission and fusion reactors, nuclear waste containment, nuclear batteries and space explorations demand materials with extraordinary thermomechanical properties and radiation resistance. Radiation can induce severe damages in materials, including swelling, hardening, creep, embrittlement and irradiation-assisted corrosion. ${ }^{1,2}$ The tolerance of radiation damage by structural materials plays a significant role in the safety and economy of nuclear energy, ${ }^{2}$ as well as the lifetime of nuclear batteries, spaceships and nuclear waste containers, as they are often exposed to long-term radiation. ${ }^{3,4}$

Nanostructuring is a key strategy to improve the radiation resistance of materials. ${ }^{5-8}$ Carbon nanotubes (CNTs) are well known to be a strong and flexible nanomaterial. If CNTs are uniformly dispersed inside metal as $1 \mathrm{D}$ fillers ${ }^{9-11}$, its high aspect ratio $\eta$ (up to $\left.10^{8}\right)^{12}$ should create prolific internal interfaces with the metal matrix that may act as venues for the radiation defects to recombine (self-heal). In addition, based on percolation theory and geometrical simulations $^{13,14}$, a random 3D network of 1D fillers can form globally percolating transport paths even with diminishing volume fraction $\phi \rightarrow 0$, if $\eta \rightarrow \infty$. 1D fillers can be efficient for this purpose, considering for example cardiovascular and plant root systems that are 1D transport networks. Helium (alpha particle) accumulation inside materials ${ }^{15}$ is a known problem that exacerbates embrittlement and swelling. ${ }^{16}$ If the 1D fillers form globally percolating paths of "nanochimneys" that can outgas the accumulated helium ${ }^{17}$ and other fission gases to an external fission-product gettering/trapping system ${ }^{18}$, they might provide an essential solution to the problem.

Key questions regarding metal-CNT composite (MCC) in the nuclear environment are: 
(i) Does the dispersion of CNTs degrade thermomechanical properties (strength, toughness, thermal conductivity ${ }^{19}$, etc.) before irradiation?

(ii) Once radiation starts, is radiation embrittlement and swelling reduced (due to self-healing effect of the filler-metal interfaces) in MCC compared to the control metal?

(iii) Even if 1D nano-fillers improve (i) and (ii), how stable are these 1D nano-fillers themselves under heavy dose of radiation? Typical radiation exposure to the nuclear fuel cladding material is 15 DPA (displacements per atom) before they are taken out of the reactor. Core internals in commercial light-water reactors should sustain around 80 DPA after 40 years of plant operations, ${ }^{20}$ and advanced fast reactors would demand even more.

In this paper we investigate the basic radiation materials science of MCC, in particular $\mathrm{Al}+\mathrm{CNT}$ composite, using a high-energy ion accelerator to inject $\mathrm{He}$ and $\mathrm{Al}$ ions which generate atomic displacements in the composite, in lieu of neutrons. We find that in addition to property improvements (i) and (ii), the 1D form factor of nano-fillers does survive up to 72 DPA of He ion irradiation, and also 72 DPA of Al self-ion radiation at room temperature, which is intriguing because every carbon and aluminum atoms are knocked out $\sim 10^{2}$ times, yet the $1 \mathrm{D}$ nanomorphologies survive, along with the prolific internal interfaces. The morphological robustness of 1D nano-fillers in non-equilibrium conditions is reminiscent of nanowire growth in chemical vapor deposition that violates equilibrium Wulff construction, and the presence of CNTs in ancient Damascus steel ${ }^{21}$ (as the equilibrium phase diagram would indicate that CNTs should be converted to blocky cementite $\mathrm{Fe}_{3} \mathrm{C}$ ).

We have synthesized Al+CNT composites, as aluminum is cheap and very widely used. Al can be used as the fuel cladding materials in research reactors, as well as containment for nuclear 
waste, components for robots in radiation environments, etc. It light density may impart significant advantage for space applications. Al has low thermal neutron absorption cross-section of 0.232 barn, above only those of $\mathrm{Mg}$ (0.063 barn), $\mathrm{Pb}(0.171$ barn) and $\mathrm{Zr}$ (0.184 barn) among structural metals, and high corrosion resistance in water, therefore it is already used widely in low-temperature research reactors. ${ }^{22}$ The development of Al+CNT may not only benefit research reactors, but also provide guidance for designing new kinds of cladding materials (e.g., $\mathrm{Zr}+\mathrm{CNT}$, Stainless-steel+CNT) that can be used in commercial reactors. Second, Al is used in nuclear battery since it is reflective, and has low production rate of Bremsstrahlung radiation due to low atomic number. Thus it has been recommended for several components in designs of nuclear battery such as shielding, current collector ${ }^{23}$ and electrode ${ }^{24} . \mathrm{Al}+\mathrm{CNT}$ will increase the lifetime of nuclear battery because of better radiation resistance. This composite may also alleviate helium accumulation from alpha decay, which is one of the main engineering issues associated with radioisotope thermoelectric generator (RTG). ${ }^{4}$

We have performed accelerator-based ion irradiation tests on Al+CNT (and pure Al control) at room temperature (homologous temperature $T / T_{\mathrm{M}}=0.32$, Al's meting point is $T_{\mathrm{M}}=933.47 \mathrm{~K}$ ). At this range, volumetric swelling from void formation becomes prominent when radiation exposure is larger than $10 \mathrm{DPA} .^{2}$

Modification of interfaces of 1D nanostructure upon irradiation plays an essential role for MCC properties. Figure 1 provides a schematic illustration of ion beam interaction with CNT. The energies of incoming ions are absorbed and transform CNT structure to rearranged carbon nanostructure, or aluminum carbide nanorods, depending on the ion type and beam energy. The 1D interfaces, if they survive, likely reduce the supersaturation of radiation-generated vacancies, 
by boosting recombination with self-interstitial atoms (SIA) and interstitial clusters. The lightweight ion irradiation generally generates more "sparse" collision cascades with lower defect density and shorter length compared to heavy ions. Therefore, He ion irradiation causes less Al/C mixing than $\mathrm{Al}$ ion irradiation since an interstitial $\mathrm{Al}$ atom can quickly find the nearest vacancy of the same chemical species. The CNT undergoes restructuring, making a helical carbon nanostructure, as shown in Fig. 1 with a yellow arrow. Irradiation with heavier Al ions, which produce "denser" collision cascades and more $\mathrm{Al} / \mathrm{C}$ mixing, ${ }^{25}$ eventually changes the composition of CNT fillers, forming an aluminum carbide phase with 1D nanorod morphology (blue arrow).

For (i),(ii), fabrication of high-quality and low-porosity composite is essential. Achieving uniform CNTs dispersion without inducing degradation to CNTs or Al matrix is the key here. Our specimen preparation consists of three steps (Fig. 2A): (step i) declustering of the CNTs on the surface of Al particles, (step ii) encapsulation of the dispersed CNTs and further consolidation into $\mathrm{Al}$ particles to form $\mathrm{Al}-\mathrm{C}$ covalent bonds by spark plasma sintering (SPS), and (step iii) hot extrusion. We used multi-walled carbon nanotubes (MWCNTs) with 10-30 nm in the diameter $D$ and $10 \mu \mathrm{m}$ in the length $L(\eta \equiv L / D=300-1000)$. The optimized processing conditions are described in detail in Supplementary Online Materials (SOM). This process is industrially scalable, and we have already produced $\mathrm{Al}+\mathrm{CNT}$ nanocomposite weighing more than $100 \mathrm{~kg}$, as shown in Fig. 2C (inset). Cost analysis indicates that its specific weight cost (including raw material cost of MWCNTs and processing costs) should be less than two times the price of bulk-scale Al alloy. The G-mode mapping from confocal Raman indicate the dispersion of CNTs in Fig. S1 A and B. Transmission electron microscopy (TEM) observation 
further verified that CNT embedded inside the Al grain as indicated by the white arrow in Fig. 2B. These observations are the evidence that CNTs were highly dispersed after the processing. A bulk specimen for ASTM E8 standard tensile testing, fabricated after hot extrusion, is used for mechanical properties testing. Typical stress-strain curves for the samples with different MWCNTs volume fraction $\phi$ are shown in Fig. 2C. The tensile strength was enhanced by $34 \%$ at 1 vol\% MWCNTs $(\phi=0.02)$, without sacrificing tensile ductility. As shown in Fig. S1C, MWCNTs strands are seen to be protruding out of the fractured area, as indicated by the white arrows. This fiber pull-out between CNTs and Al induces load transfer and improves fracture toughness. ${ }^{26}$

To test the radiation tolerance of the $\mathrm{Al}+\mathrm{CNT}$ composite, the sample was irradiated by 100 $\mathrm{keV}$ helium ions and $2 \mathrm{MeV}$ aluminum self-ion up to 3.6, 16 and 72 DPA (see SOM), respectively. The results were compared with the pure Al control samples under the same irradiation conditions. The diameter of the inner space and the wall thickness of the MWCNT are $10 \mathrm{~nm}$ and 7 10 nm, respectively, as indicated in the TEM image in Fig. 3A. The initial geometry does resemble a "nano-chimney". The graphene walls of the CNTs were clearly visible in the TEM images shown in Fig. 3B and C, indicating no significant chemical mixing the CNTs. If the MWCNTs are entirely straight and randomly distributed, then analytical modeling and Monte Carlo simulations gives percolation threshold estimate ${ }^{13,14}$ :

$$
\phi_{\mathrm{c}} \approx \frac{1}{2 \frac{L}{D}+3+\pi+\frac{\pi}{2} \frac{D}{L}}
$$


which for aspect ratio $\eta \equiv L / D=300$, gives $\phi_{c}=0.0016$, and for $\eta \equiv L / D=1000$, gives $\phi_{c}=5 \times 10^{-4}$. The MWCNT volume fraction we have here is an order of magnitude larger than $\phi_{c}$, therefore the MWCNTs should form a globally percolating network of nano-chimneys. Helium gas is expected to travel facilely in 1D hollow structures like MWCNTs with smooth interior walls. ${ }^{17}$

Figures 3D and E show the control Al samples after 3.6 DPA He-ion irradiation and 72 DPA Al self-ion irradiation, respectively. The irradiation generates nanocavities inside by the aggregation of radiation-induced vacancies, and the positive He gas pressure further stabilizes the bigger cavities compared to Al-ion irradiation. Bubbles appear at just 3.6 DPA in pure Al for He-ion irradiation. The formation of large cavities with diameters ranging 100 200 nm was observed in the control $\mathrm{Al}$ (Fig. 3D left). The higher magnification indicates that small cavities were also generated (Fig. 3D right). In contrast, the $\mathrm{Al}+\mathrm{CNT} 1$ vol\% sample has no cavity generation at the same DPA (Fig. 3E). The higher magnification provides clear evidence of no bubble/void generation at 3.6 DPA He-ion irradiation (Fig. 3F) in Al+CNT. Furthermore, no cavity was observed even after 72 DPA Al self-ion irradiation of the Al+CNT (Fig. 3G). CNTs dispersed inside $\mathrm{Al}$ grain seem to suppress cavity generation completely up to at least 3.6 DPA for He-ion and 72 DPA for Al self-ion radiation, and the answer to (ii) should be positive from the structural point of view.

He-ion radiation to 72 DPA was further carried out to study severe radiation damage condition. Large cavities about $500 \mathrm{~nm}$ in diameter were observed in $\mathrm{Al}$ without CNTs (Fig. 4A and S3A). The surface indicates obvious surface cracking occurred from the volume expansion of the cavities after the irradiation (Fig. S2A, bottom). Cavities are also generated in Al+CNT 1 vol\% sample at 72 DPA He-ion irradiation, but much smaller than those of control Al. (Fig. 4B and 
$\mathrm{S3B})$. The largest cavity is $170 \mathrm{~nm}$ in diameter, 20 times smaller in volume than the pore in the control Al. This suggests that the incorporation of MWCNTs in Al suppresses porosity development in severe radiation damage conditions. This obvious reduction of porosity in AlCNTs composite implies that $\mathrm{He}$ gas diffused out of Al matrix robustly. Two mechanisms are possible: i) He gas diffused out along the CNT-metal interface, or ii) the interspace and central hollow space inside CNTs acts as 'nano-chimneys' for diffusion of He gas. Since the mechanical strength is enhanced significantly by load transfer associated with strong anchoring of $\mathrm{Al}$ onto the CNT surface ${ }^{9,27}$, the possibility of the former is small. Therefore, we believe that the globally percolating "nano-chimney" network plays a role for He outgassing.

To quantify the effect of carbon on the radiation damage induced by He ion irradiation in the $\mathrm{Al}$, the stopping and range of ions in matter (SRIM-2013) simulation [srim.org] was performed with/without carbon element in the $\mathrm{Al}$ matrix. The carbon content of $\mathrm{Al}+1 \mathrm{vol} \% \mathrm{CNT}$ was roughly $0.5 \mathrm{wt} \%$. In the simulation, we uniformly dispersed carbon atoms in the $\mathrm{Al}$ matrix to extract the effect of the carbon atoms alone. The maximum DPA is predicted to occur at $534 \mathrm{~nm}$ in depth, slightly shallower than the maximum peak $(596 \mathrm{~nm})$ of injected He ion. Exactly the same DPA profiles were observed regardless of the presence of carbon, as shown in Fig. S4. The $0.5 \mathrm{wt} \%$ carbon in $\mathrm{Al}$ hence has negligible influence on the helium injection and DPA profiles. Figure $3 \mathrm{C}$ shows the relationship between the injected ion/pore generations versus the depth. The simulated damage profiles agree well with the experimentally observed porosity generation profile. However, the absolute cavity area and the size are significantly smaller in the Al+CNT composites than in the control sample. This suggests that the MWCNTs giving high internal interface area is key to the reduced porosity creation. More detailed modeling including the 
shapes of the MWCNT inclusion and the CNT-Al interactions is necessary to precisely quantify the structural effect, which is beyond the scope of this paper.

If the MWCNTs are randomly dispersed, then the furthest distance between any point of its nearest MWCNTs scales as $L_{\text {furthest }} \propto D \phi^{-1 / 2}$ (D=diameter). For our 1vol\% MWCNT sample, $L_{\text {furthest }}$ should be around $200 \mathrm{~nm}$. This is still an order of magnitude longer than the typical size of a radiation cascade, which is $10-20 \mathrm{~nm}$, therefore the improvement in porosity suggests that porosity development involves length scales quite beyond a single cascade annealing. For comparison, ultra-fine grained austenitic stainless steel with a grain size of $100 \mathrm{~nm}$ was recently shown to exhibit 5 times slower void swelling rate up to $80 \mathrm{DPA},{ }^{7}$ and $L_{\text {furthest }}$ in that case should be around $50 \mathrm{~nm}$ if all the grain boundaries (GB) are effective venues for recombination. Compared to that system of "2D nanoengineered" network of GBs ${ }^{7}$, our "1D nanoengineered" CNTs/Al has 4 times longer $L_{\text {furthest }}$ and 15 times less interfacial area per volume. Yet our system seems to be still similarly effective in cavity suppression.

The above demonstrates aplenty that $\mathrm{Al}+\mathrm{CNT}$ composite was successful in reducing the structural damage. To show that it leads to property improvement, we conducted micro hardness test to evaluate the change in strength of $\mathrm{Al}+\mathrm{CNT}$ under radiation exposure. Since the irradiation damage from the ion accelerator was localized beneath the surface within $1 \mu \mathrm{m}$ depth, we selected the Knoop micro-hardness test to quantify the mechanical behavior in the damaged region. The Knoop micro-hardness test is specially designed for thin film samples. Cracks and porous structure under the surface were observed in the control Al after the Knoop indentation, whereas $\mathrm{Al}+\mathrm{CNT}$ sample showed almost no cracks, as seen in Fig. $4 \mathrm{D}$ and E, indicating that the $\mathrm{Al}+\mathrm{CNT}$ sample has less irradiation embrittlement and swelling. The hardness value further 
verify this observation. The hardness change was measured as a function of DPA as shown in Fig. 4F. Note that the hardness increased up to $328 \mathrm{HK}$ at 3.6 DPA in the control Al. In contrast, our Al+CNT nanocomposite, even though it starts out having higher hardness by virtue of higher strength (i), hardens much less compared to control Al (ii). The initial radiation hardening observed in metallic materials results from the obstacles to dislocations, such as point-defect clusters, stacking fault tetrahedra and cavities, generated by radiation. Thus, we again verifies that our " $1 \mathrm{D}$ nanoengineered" $\mathrm{Al}+\mathrm{CNT}$ has better radiation tolerance (specifically radiation hardening and embrittlement) compared to the reference control Al.

However, once above 3.6 DPA, the Knoop hardness of control Al decreased with increasing helium ion irradiation dose. This phenomenon could be explained by the severe porosity development which reduced the apparent density of materials. The cavity volume fraction in control Al reached $25 \%$ at $72 \mathrm{DPA}$ (Fig. 4A). The increasing volume of pores cause the transition from hardening to softening, ${ }^{28}$ and will result in exceptionally poor toughness as tensile fracture is very sensitive to the size of the largest flaw. In contrast, the cavity volume fraction reached only $4.7 \%$ for $\mathrm{Al}+\mathrm{CNT}$ at $72 \mathrm{DPA}$, with the largest pore 20 times smaller in volume (Figs S3 A and B). Also, the maximum value of the hardness in Al+CNT was reached at 16 DPA (5 times larger dose than control Al), and the $240 \mathrm{HK}$ peak hardening value was much lower than that of the control Al. We are thus confident that the mechanical properties of $\mathrm{Al}+\mathrm{CNT}$ is more tolerant of both low and high doses of radiation.

High-resolution TEM (HRTEM) was performed on the post-irradiated Al+CNT, as shown in Fig. $5 \mathrm{~A}$ and B. Several tubular cross-sectional structures near each pore were observed (Fig. 5A). The tubular structure is still retained after 72 DPA He-ion radiation. Some of the tubular walls 
merged with each other and the helical shapes were also found, as shown in Fig. $1^{29}$. Thus, the 1D nano-fillers maintain its general tubular morphology under the He ion irradiation (which generates sparser cascades). Raman spectroscopy indicates quite drastic changes in atomic bonding inside the tubules at higher DPA He-ion irradiation, as confirmed from Raman spectra of $\mathrm{D}$ and $\mathrm{G}$ bands in Fig. 5C. The strong signal near $1440 \mathrm{~cm}^{-1}$ corresponds to tetrahedral amorphous carbon (ta-C) with highest $\mathrm{sp}^{3}$ content $(80-90 \%)^{30}$. Electron energy loss spectroscopy (EELS) mapping in TEM shows the region with a high carbon concentration (20 nm in width) corresponding to the original diameter of the CNT (Fig. 3A and Fig. S6B). The $\mathrm{sp}^{3} / \mathrm{sp}^{2}$ mapping results (Fig. S6 C and D) indicate strong $\mathrm{sp}^{3}$ signal at the region of high carbon concentration (see SOM for detail). The observations suggest that the carbon tubular nanostructures observed in TEM are composed of diamond-like carbon with tetrahedral amorphous $\mathrm{sp}^{3}$ bonding, instead of aluminum carbide $\left(\mathrm{Al}_{4} \mathrm{C}_{3}\right)$ which should form according to the equilibrium phase diagram below $2160^{\circ} \mathrm{C}^{31}$

In reference to pure $\mathrm{Al}$ and graphite, the Gibbs free energy of formation for the stable phase of $\mathrm{Al}_{4} \mathrm{C}_{3}$ (rhombohedral) is $-194.4 \mathrm{~kJ} / \mathrm{mol}$ at room temperature ${ }^{31}$ or $-2.01 \mathrm{eV}$ per $\mathrm{Al}_{4} \mathrm{C}_{3}$ formula unit. On a per carbon basis, it is not as high as $\mathrm{ZrC}\left(-2.14 \mathrm{eV}\right.$ per $\left.\mathrm{ZrC}^{32}\right)$, but is comparable to $\mathrm{SiC}$ ($0.76 \mathrm{eV}$ per $\mathrm{SiC})$ and much higher than cementite $\left(-0.18 \mathrm{eV}\right.$ per $\left.\mathrm{Fe}_{3} \mathrm{C}\right)$. So the fact that much of the carbon nanostructures survive without forming the carbide after 72 DPA He-ion irradiation is somewhat surprising. On the other hand, the conversion of $\mathrm{sp}^{2}$ bonding of carbon in CNTs to $\mathrm{sp}^{3}$ of ta-C agrees with the previous understanding of radiation damage of carbon. ${ }^{33}$

Aluminum self-ion irradiation with higher energy of $2 \mathrm{MeV}$ (20x that of helium ion) which create denser cascades ${ }^{25}$ eventually disintegrates the pure carbon nanostructure, and generates 
slender $\mathrm{Al}_{4} \mathrm{C}_{3}$ nanocarbides, as shown in Figs. 5D and S8, and illustrated in Fig. 1. The denser cascade provides higher probability to mix carbon with the matrix aluminum atoms. The $1 \mathrm{D}$ nature of $\mathrm{Al}_{4} \mathrm{C}_{3}$ nanocarbides was confirmed in a series of tilting images inside the TEM. The electron diffraction along $\mathrm{Al}$ [001] zone axis on the nanocarbide shows that the new structure embedded in the matrix is not the rhombohedral phase of $\mathrm{Al}_{4} \mathrm{C}_{3}$ (ICSD number 14397), but a metastable triclinic phase (materialsproject.org mp632442). Density functional theory calculations reveal that, intriguingly, this metastable $\mathrm{Al}_{4} \mathrm{C}_{3}$ nanocarbide has higher formation energy of about $2.8 \mathrm{eV}$ per unit formula above the rhombohedral phase ground state. This energetic metastability is about $1.877 \mathrm{MJ} / \mathrm{kg}$, almost half of the detonation energy density of TNT. We have also determined that many distinct lattice orientation relationships are present between the newly formed $\mathrm{Al}_{4} \mathrm{C}_{3}$ and $\mathrm{Al}$ matrix, with semicoherent and incoherent interfaces based on high-resolution TEM observations. The 1D nanocarbides likely benefit energetically from the interfacial energy considerations with the matrix, which otherwise would be considered high energy in bulk form. Fig. 5D is quite remarkable in that it shows two $\mathrm{Al}_{4} \mathrm{C}_{3}$ nanocarbides running parallel to each other, separated by $\sim 20 \mathrm{~nm}$, on the order of $D$ of the original MWCNTs. We surmise these two nanocarbides are decomposition products from the same MWCNT, that originally ran in the same direction, like "fly in amber". The high-energy self-ion radiation destroyed the hollowness of the MWCNT and backfilled it with Al, but vestiges of the original 1D nanostructures remain like fossil record. The nanocarbides are thus templated by the original carbon nanostructures, and this in situ formation could be a new paradigm for creating radiationtolerant nanodispersion-strengthened metals. 
In summary, we can mass-produce Al-CNT nanocomposite cheaply, at $100 \mathrm{~kg}$ scale and at no more than $2 \times$ the cost. With regard to question (i), CNTs improve strength while maintaining tensile ductility. Our helium and aluminum ion irradiation experiments demonstrate that uniform dispersion of CNT reduces radiation hardening and embrittlement. These evidences indicate that the answer to (ii) is affirmative, due to efficient defect recombination at the incoherent CNTmetal interfaces. Detailed microstructural characterizations further demonstrate that the prolific 1D slender form factors are surprisingly robust under radiation, and survive up to 72 DPA of Heion and Al-ion irradiations, answering question (iii). Therefore, Al-CNT nanocomposite satisfies all three main concerns (i), (ii) and (iii), providing a paradigm to improve components in nuclear fission and fusion reactors, nuclear waste containment, nuclear batteries and space explorations that demand materials with extraordinary thermomechanical properties and radiation resistance.

\section{Acknowledgements}

We acknowledge support by NSF DMR-1410636 and DMR-1120901, and US Department of Energy, Office of Basic Energy Sciences, under Grant No. DE-SC0006725. This research was also supported by institute for Basic Science (IBS-R011-D1) and Basic Science Research Program through the National Research Foundation of Korea (NRF) funded by the Ministry of Education, Science and Technology (NRF-2013R1A6A3A03064138). EMB thanks support from PICT0092 and a SeCTyP-UNCuyo grant. RG and MK thanks the support from Fondo Nacional de Investigaciones Cientificas y Tecnologicas (FONDECYT, Chile) under grants \#3140526 (RG), \#1120399 and 1130272 (MK), and Center for the Development of Nanoscience and Nanotechnology CEDENNA FB0807 (RG and MK) 
Figure legends:

Fig. 1.I Schematic illustration of shape changes on CNT, recombination, and helium outgas. Under ion irradiation, the disintegration of CNT and formation of aluminum carbide (blue arrow) from high energy ion and restructuring to helical CNT structure (yellow arrow) from low energy ion are indicated.

Fig. 2.I Fabrication process and microstructure/mechanical properties of Al+CNT composites. (A) A schematic representation for the fabrication of $\mathrm{Al}+\mathrm{CNT}$ composite. (B) Dispersion of CNT inside Al grain in TEM, (C) Stress-strain curve (inset: $100 \mathrm{~kg}$ of the Al+CNT composite). Dispersion of CNTs in grain improves the tensile strength without sacrificing ductility.

Fig. 3. I Structural evolution of Al+CNT composite under ion irradiation. TEM image of (A) pristine CNT, (B) and (C) intact wall structure of CNT in Al matrix. Microstructure of (D) control $\mathrm{Al}$ and (E) Al+CNT after helium ion irradiation at 3.6 DPA, (F) control Al and (G) 
$\mathrm{Al}+\mathrm{CNT}$ after aluminum self-ion irradiation at $72 \mathrm{DPA}$. Note, no pores were generated by dispersing the 1 vol\% of CNT in $\mathrm{Al}$ matrix in $(\mathrm{E})$ and $(\mathrm{G})$.

Fig. 4.I Quanitfication and mechanical responsse of pore generation after 72 DPA helium ion irradiation. SEM image of (A) highly porous control $\mathrm{Al}$ and (B) $\mathrm{Al}+\mathrm{CNT} 1$ vol\%. (C) Injected ion (SRIM) and pore areas versus depth. SRIM is exerted by He ion injection with same experimental parameter. Indented area observation on (D) control $\mathrm{Al}$ and (E) $\mathrm{Al}+\mathrm{CNT}$ composites. Note, highly cracked and porous structure are observed near indented areas in control Al, implying brittle and pore under the surface. (F) Knoop hardness versus DPA.

Fig. 5.I Structure of CNT after 72 DPA irradiation. (A) Traces and (B) wall structure of CNTs after helium ion irradiation, and (C) Raman spectrum at different DPA. (D) $\mathrm{Al}_{4} \mathrm{C}_{3}$ nanocarbide under $72 \mathrm{DPA} \mathrm{Al}$ self-ion irradiation. Note: the structure of $\mathrm{Al}_{4} \mathrm{C}_{3}$ nanocarbide is described in supplementary (Figs. S8). 


\title{
References and Notes:
}

\author{
http://li.mit.edu/S/dc/Paper/
}

1 Bringa, E. M. et al. Are Nanoporous Materials Radiation Resistant? Nano Letters 12, 3351-3355, doi:10.1021/nl201383u (2012).

2 Zinkle, S. J. \& Busby, J. T. Structural materials for fission \& fusion energy. Mater Today 12, 12-19 (2009).

3 Novikov, L. S. et al. Radiation effects on spacecraft materials. J Surf Investig-X-Ra 3, 199-214, doi:10.1134/S1027451009020062 (2009).

4 Wacharasindhu, T., Kwon, J. W., Meier, D. E. \& Robertson, J. D. Radioisotope microbattery based on liquid semiconductor. Appl Phys Lett 95, doi:Artn 014103

10.1063/1.3160542 (2009).

5 Beyerlein, I. J. et al. Radiation damage tolerant nanomaterials. Mater Today 16, 443-449, doi:10.1016/j.mattod.2013.10.019 (2013).

6 Wurster, S. \& Pippan, R. Nanostructured metals under irradiation. Scripta Mater 60, 1083-1087, doi:10.1016/j.scriptamat.2009.01.011 (2009).

7 Sun, C. et al. Superior radiation-resistant nanoengineered austenitic 304L stainless steel for applications in extreme radiation environments. Sci. Rep. 5, 7801, doi:10.1038/srep07801

http://www.nature.com/srep/2015/150115/srep07801/abs/srep07801.htm|\#supplementaryinformation (2015).

8 Odette, G. R. Recent Progress in Developing and Qualifying Nanostructured Ferritic Alloys for Advanced Fission and Fusion Applications. Jom-Us 66, 2427-2441, doi:10.1007/s11837-0141207-5 (2014).

9 So, K. P. et al. Improving the wettability of aluminum on carbon nanotubes. Acta Materialia 59, 3313-3320, doi:10.1016/j.actamat.2011.01.061 (2011).

10 So, K. P. et al. SiC formation on carbon nanotube surface for improving wettability with aluminum. Compos Sci Technol 74, 6-13, doi:10.1016/j.compscitech.2012.09.014 (2013).

11 Bakshi, S. R., Lahiri, D. \& Agarwal, A. Carbon nanotube reinforced metal matrix composites - a review. International Materials Reviews 55, 41-64, doi:10.1179/095066009X12572530170543 (2010).

12 Wang, X. et al. Fabrication of Ultralong and Electrically Uniform Single-Walled Carbon Nanotubes on Clean Substrates. Nano Letters 9, 3137-3141, doi:10.1021/nl901260b (2009).

13 White, S. I. et al. Electrical Percolation Behavior in Silver Nanowire-Polystyrene Composites: Simulation and Experiment. Adv Funct Mater 20, 2709-2716, doi:10.1002/adfm.201000451 (2010).

14 Mutiso, R. M., Sherrott, M. C., Li, J. \& Winey, K. I. Simulations and generalized model of the effect of filler size dispersity on electrical percolation in rod networks (vol 86, 214306, 2012). Phys Rev B 87, doi:Artn 019902

10.1103/Physrevb.87.019902 (2013).

15 Kashinath, A., Misra, A. \& Demkowicz, M. J. Stable Storage of Helium in Nanoscale Platelets at Semicoherent Interfaces. Phys Rev Lett 110, 086101 (2013).

16 Odette, G. R., Alinger, M. J. \& Wirth, B. D. Recent developments in irradiation-resistant steels. Annu Rev Mater Res 38, 471-503, doi:10.1146/annurev.matsci.38.060407.130315 (2008). 
17 Verweij, H., Schillo, M. C. \& Li, J. Fast Mass Transport Through Carbon Nanotube Membranes. Small 3, 1996-2004, doi:10.1002/smll.200700368 (2007).

18 Schwartz, A. et al. Postirradiation Examination of Peach Bottom Fuel Elements E05-05 and C0505 and Related Analyses

(Gulf General Atomic, U. S. Atomic Energy Commission 1969).

$19 \mathrm{Li}, \mathrm{J} .$, Porter, L. \& Yip, S. Atomistic modeling of finite-temperature properties of crystalline betaSiC - II. Thermal conductivity and effects of point defects. J. Nucl. Mater. 255, 139-152, doi:10.1016/s0022-3115(98)00034-8 (1998).

20 Zinkle, S. J. \& Was, G. S. Materials challenges in nuclear energy. Acta Materialia 61, 735-758, doi:http://dx.doi.org/10.1016/j.actamat.2012.11.004 (2013).

21 Reibold, M. et al. Materials: Carbon nanotubes in an ancient Damascus sabre. Nature 444, 286286, doi:http://www.nature.com/nature/journal/v444/n7117/suppinfo/444286a S1.html (2006).

22 Farrell, K. Performance of Aluminum in Research Reactors. Comprehensive Nuclear Materials, Vol 5: Material Performance and Corrosion/Waste Materials, 143-175 (2012).

(Google Patents, 1958).

24 Shanks, H. R. (Google Patents, 1998).

25 Rafael I. Gonzalez et al. Metal-Nanotube composites as radiation resistant materials. to be submitted (2015).

26 Boesl, B., Lahiri, D., Behdad, S. \& Agarwal, A. Direct observation of carbon nanotube induced strengthening in aluminum composite via in situ tensile tests. Carbon 69, 79-85, doi:http://dx.doi.org/10.1016/i.carbon.2013.11.061 (2014).

27 So, K. P. et al. Prevention of ductility reduction in CNT-reinforced aluminum via multi-step rupturing process. (2016).

28 Ashby, M. F. \& Medalist, R. F. M. The mechanical properties of cellular solids. MTA 14, 17551769, doi:10.1007/bf02645546 (1983).

29 Terrones, M. et al. Molecular Junctions by Joining Single-Walled Carbon Nanotubes. Physical Review Letters 89, 075505 (2002).

30 Ferrari, A. C. \& Robertson, J. Raman spectroscopy of amorphous, nanostructured, diamond-like carbon, and nanodiamond. Phil. Trans. R. Soc. Lond. A 362, 2477-2512, doi:10.1098/rsta.2004.1452 (2004).

31 Qiu, C. \& Metselaar, R. Solubility of carbon in liquid Al and stability of Al4C3. Journal of Alloys and Compounds 216, 55-60, doi:http://dx.doi.org/10.1016/0925-8388(94)91042-1 (1994).

32 Li, J., Liao, D., Yip, S., Najafabadi, R. \& Ecker, L. Force-based many-body interatomic potential for ZrC. Journal of Applied Physics 93, 9072-9085, doi:doi:http://dx.doi.org/10.1063/1.1567819 (2003).

33 Banhart, F. Irradiation effects in carbon nanostructures. Reports on Progress in Physics 62, 11811221, doi:10.1088/0034-4885/62/8/201 (1999). 


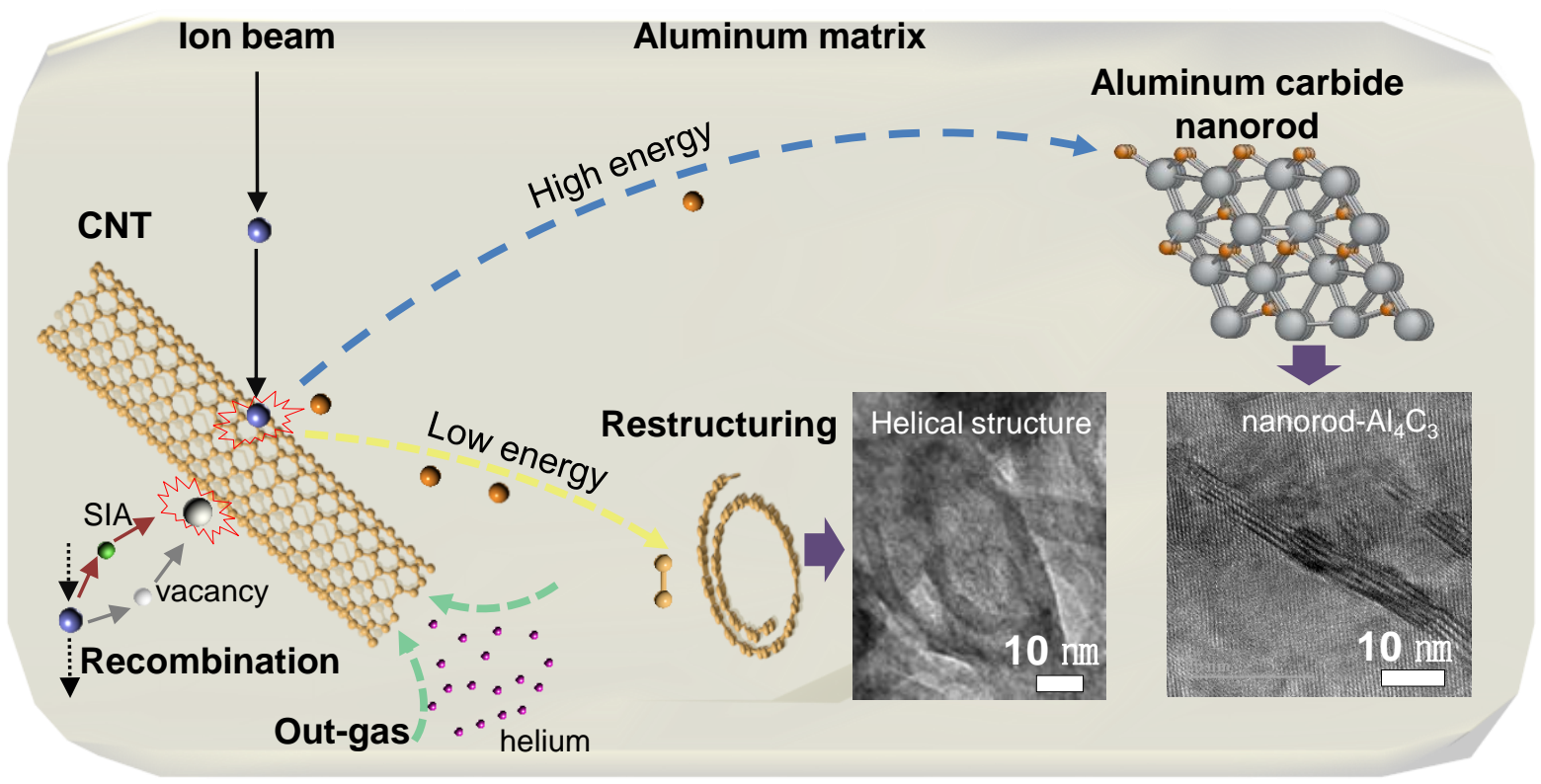

Fig. 1. 

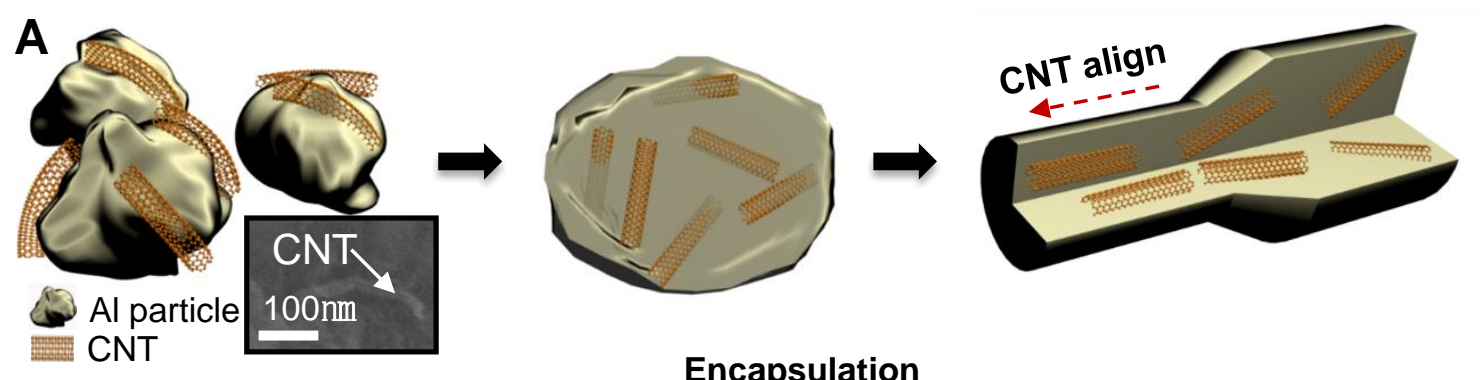

Step i : Declustered CNTs

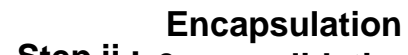

Step iii : Extrusion
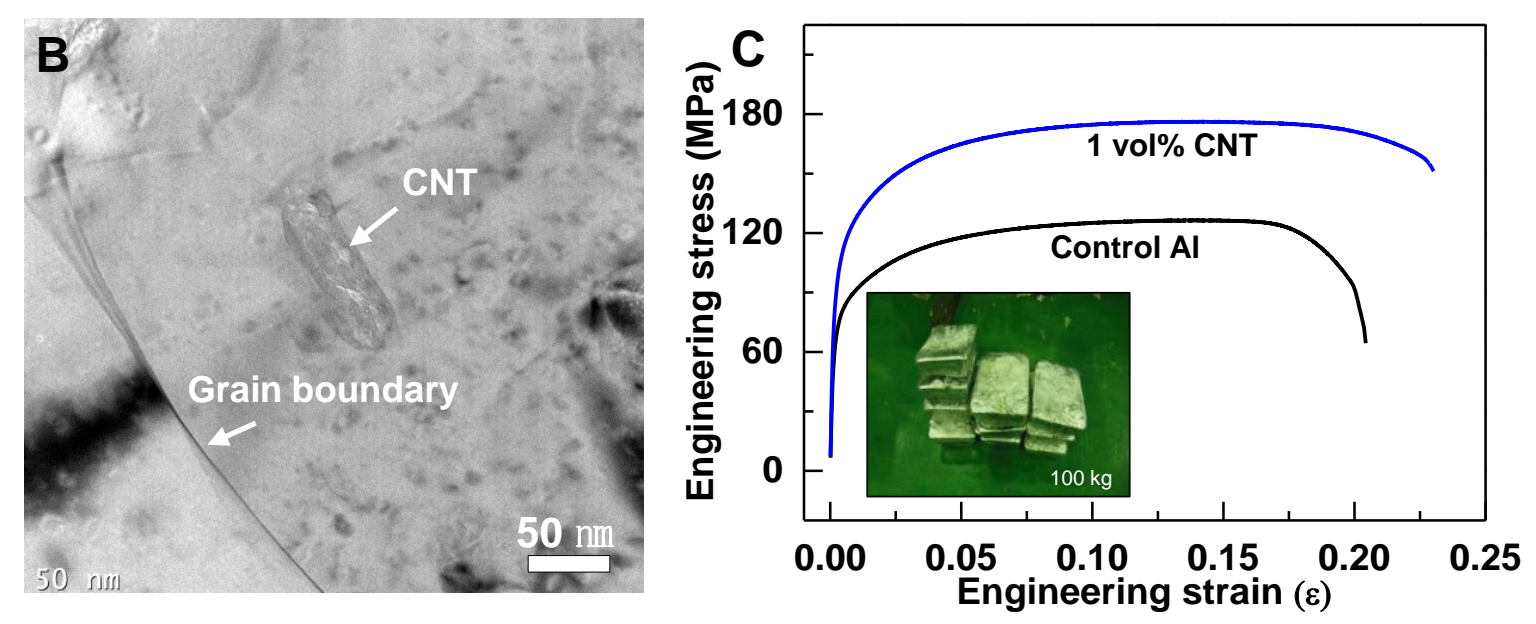

Fig. 2. 
As-prepared Al/CNT
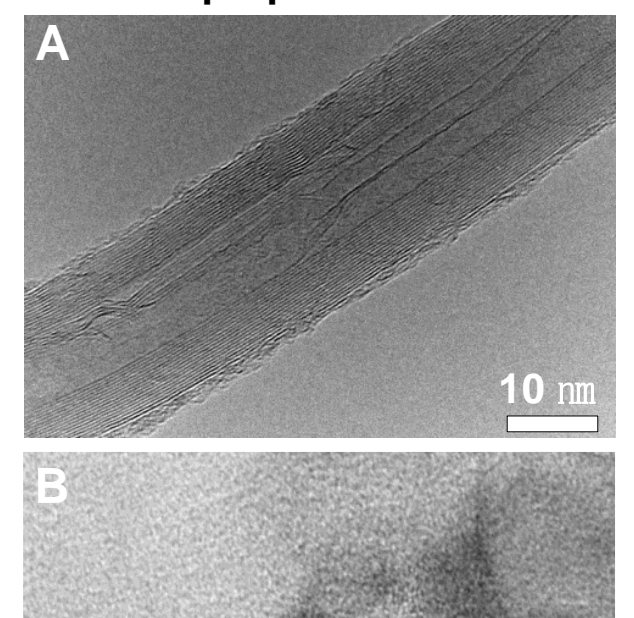

eNT
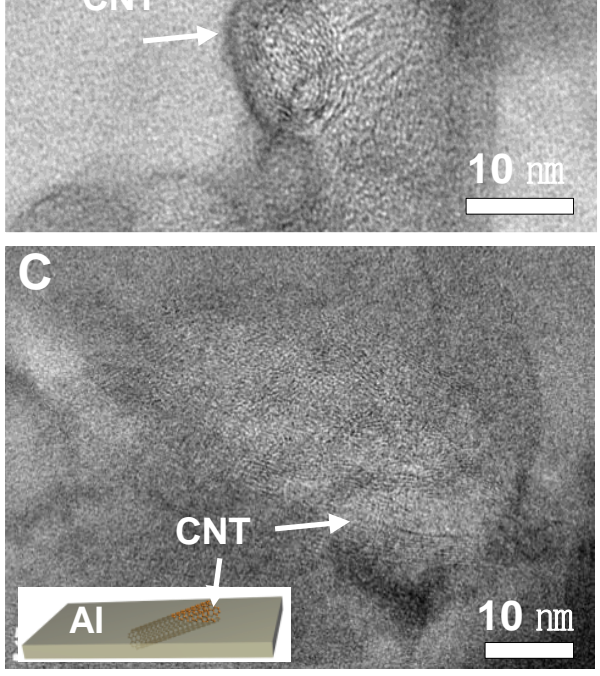

He-ion irradiation (3.6 DPA)

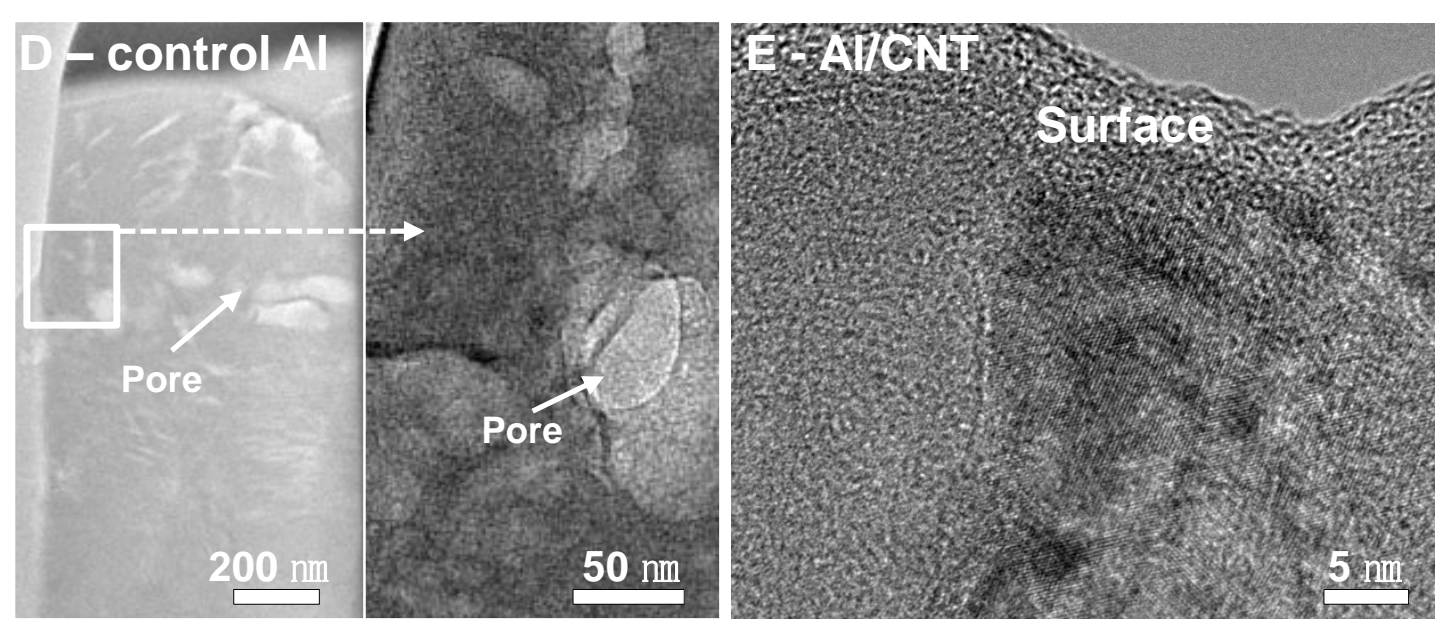

\section{Al self-ion irradiation (72 DPA)}

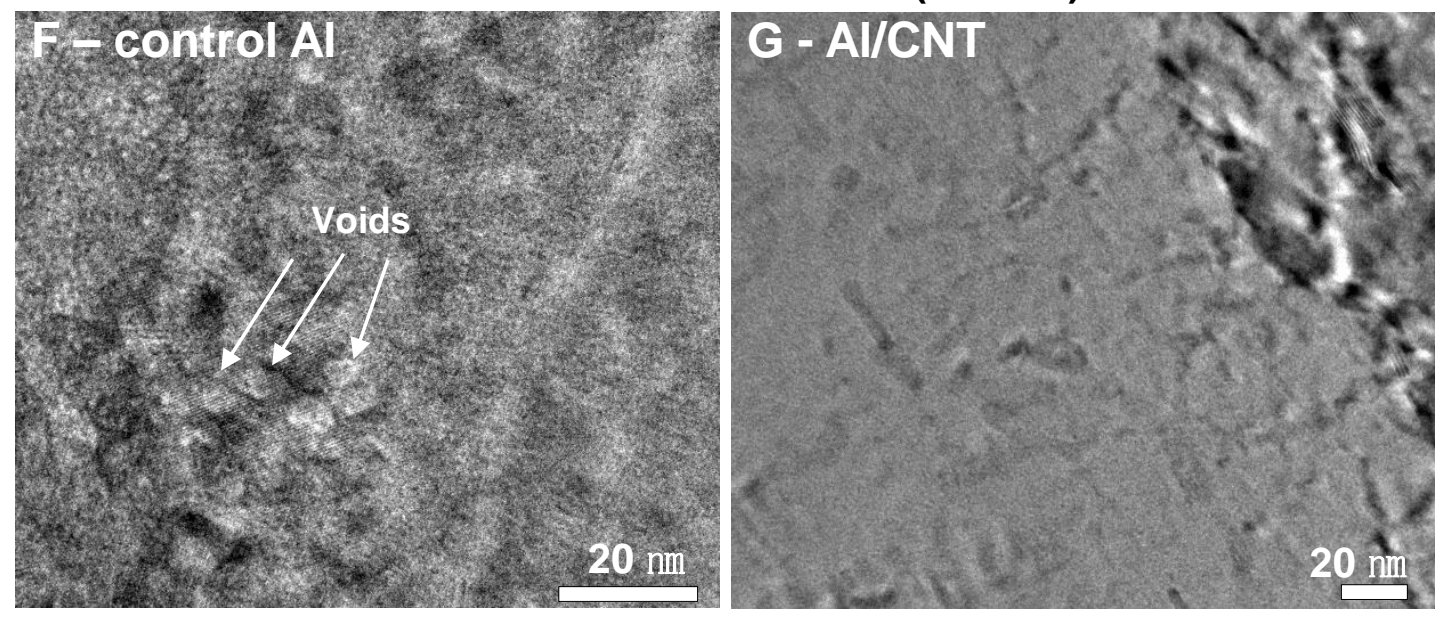

Fig. 3. 

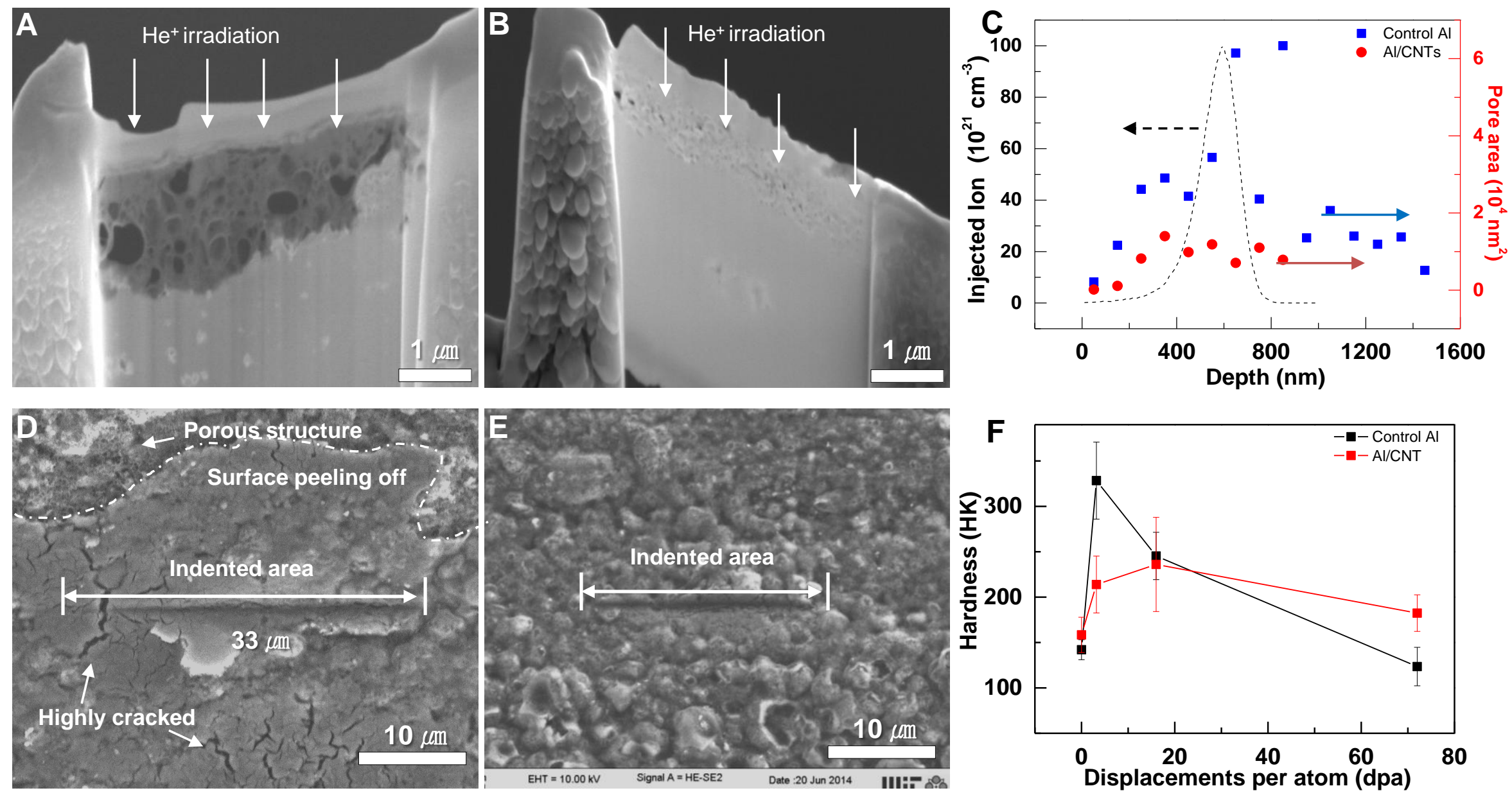

Fig. 4. 

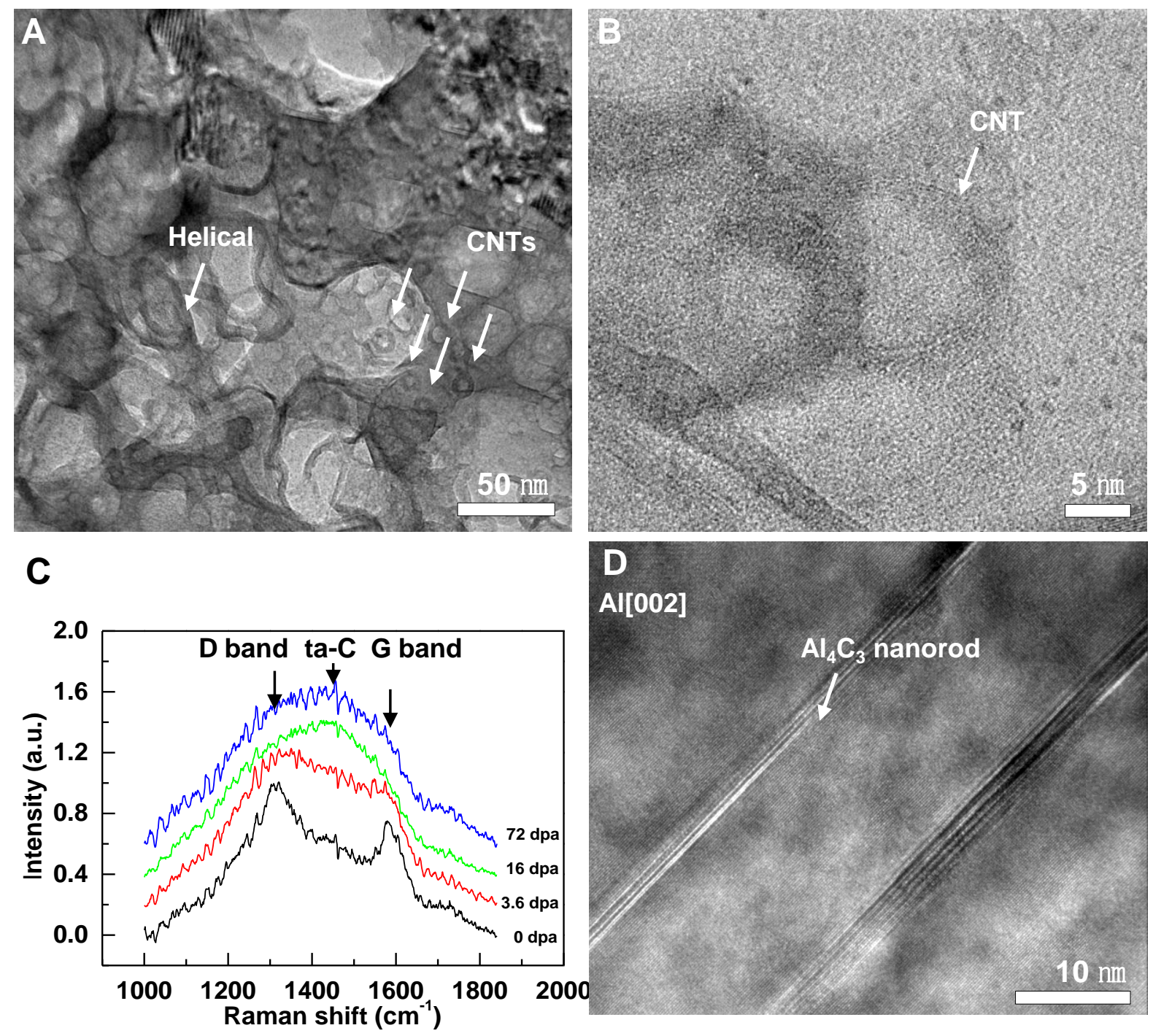

Fig. 5. 

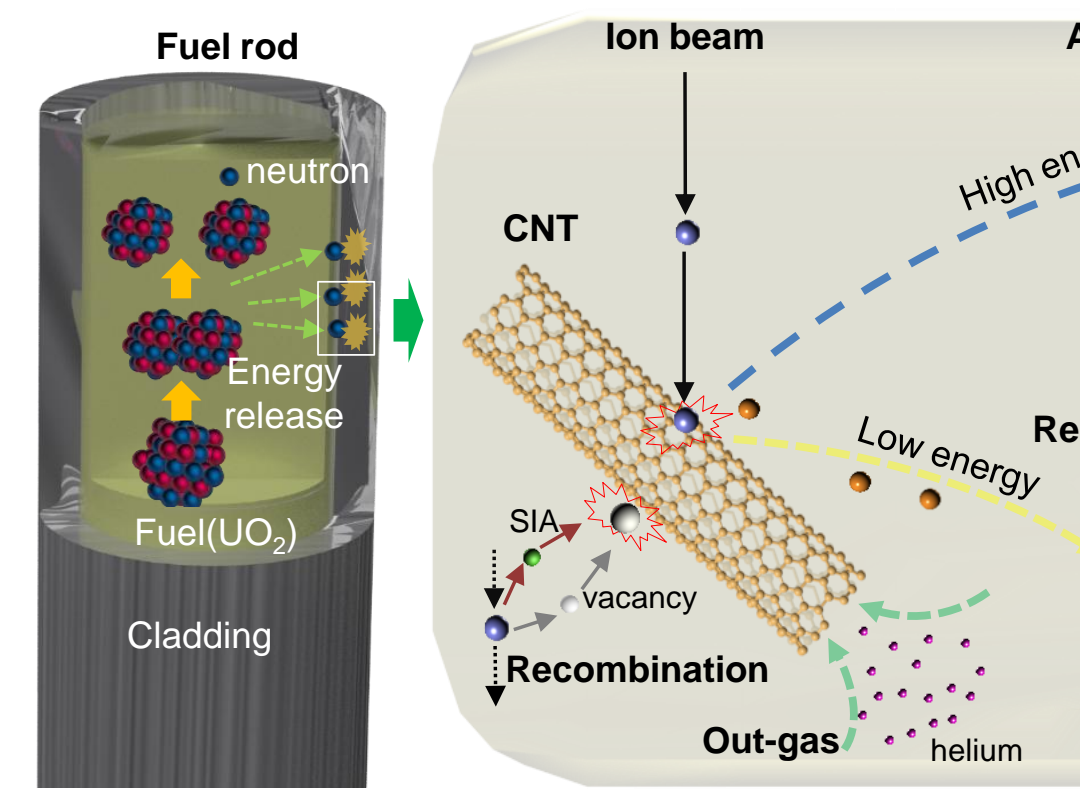

\section{Aluminum matrix}

helium 


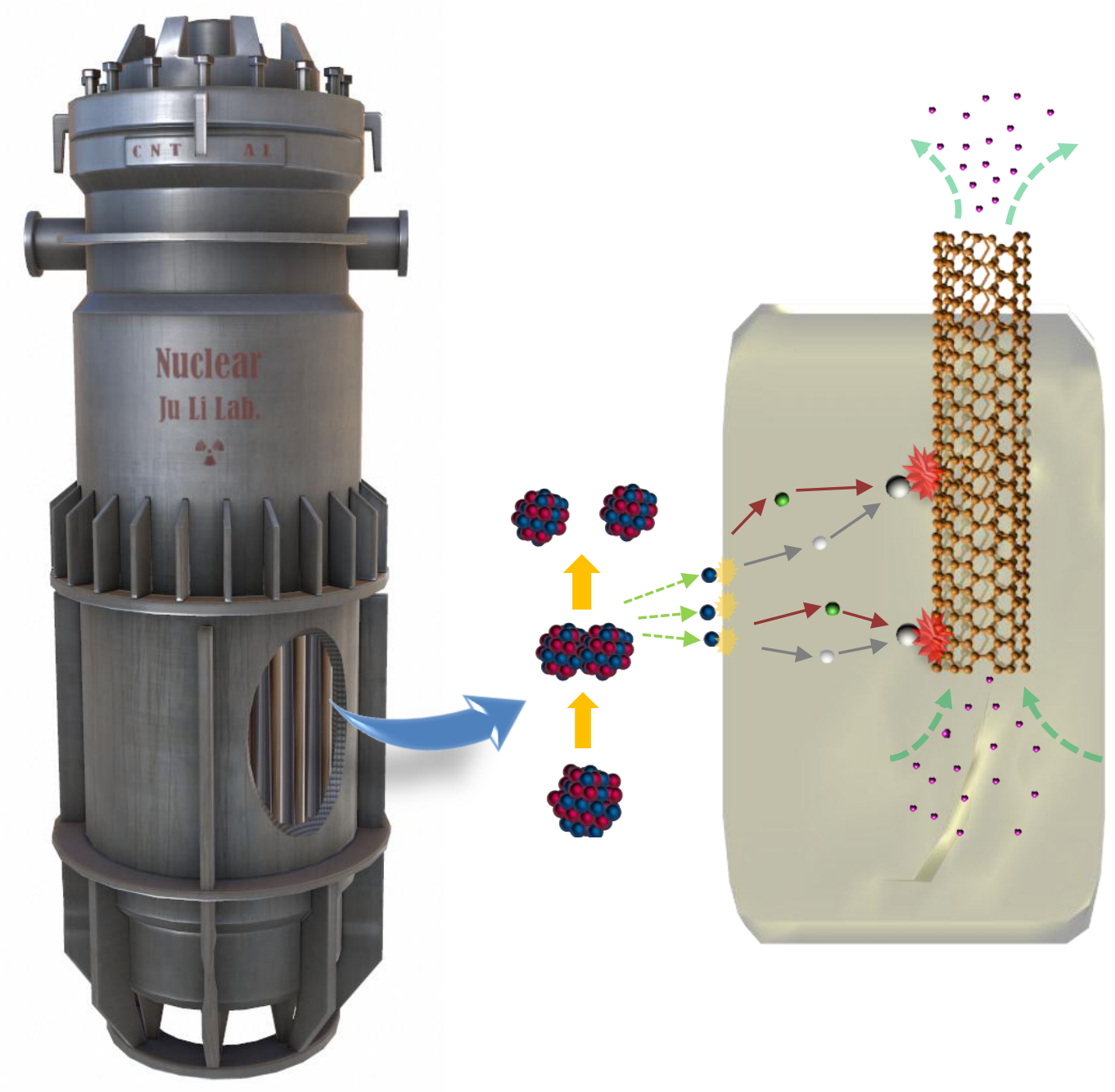

